\title{
Numerical Representation of MHD Turbulence Prior to the Ultimate Phase of Decay
}

\author{
Ripan Roy*, M. Abu Bkar Pk \\ Department of Applied Mathematics, University of Rajshahi, Rajshahi, Bangladesh
}

Email address:

ripan.ru.bd@gmail.com (R. Roy), abubakarpk_ru@yahoo.com (M. A. B. Pk)

${ }^{*}$ Corresponding author

\section{To cite this article:}

Ripan Roy, M. Abu Bkar Pk. Numerical Representation of MHD Turbulence Prior to the Ultimate Phase of Decay. Pure and Applied Mathematics Journal. Vol. 5, No. 2, 2016, pp. 32-38. doi: 10.11648/j.pamj.20160502.11

Received: February 26, 2016; Accepted: March 8, 2016; Published: March 21, 2016

\begin{abstract}
Following Deissler's approach the magnetic field fluctuation in MHD turbulence prior to the ultimatephase of decay is studied. Two and three point correlation equations have been obtained and the set of equations is made determinate by neglecting the quadruple correlations in comparison with second and third order correlations. The correlation equations are changed to spectral form by taking their Fourier transforms. The decay law for magnetic field fluctuations is obtained and discussed the problem numerically and represented the results graphically.
\end{abstract}

Keywords: Correlation Function, Deissler's Method, Fourier-Transformation, Matlab, Navier-Stokes Equation

\section{Introduction}

Magneto hydrodynamic (MHD) turbulence is characterized by nonlinear interactions among fluctuations of the magnetic field and flow velocity over a range of spatial and temporal scales. It plays an important role in the transport of energetic particles. Magneto hydrodynamics turbulence has been employed as a physical model for a wide range of applications in astrophysical and space plasma physics. The fundamental aspects of MHD turbulence include spectral energy transfer, non-locality, and anisotropy, each of which is related to the multiplicity of dynamical time scales that may be present. These basic issues can be discussed based on the concepts of magnetic Prandtle number of the small scales in the magnetic field. The magnetic Prandtle number defined as the ratio between the kinematic viscosity and the magnetic diffusivity. Boyd (2001) discussed Chebyshev and Forier spectral methods. Shebalin (2002) explained the statistical mechanics of ideal homogeneous turbulence. Biskamp (2003) obtained magneto hydrodynamic turbulence. Islam and Sarker (2001) developed the first order reactant in MHD turbulence before the final period of decay for the case of multi-point and multi-time. Shebalin (2006) also oriented ideal homogeneous magnetohydrodynamic turbulence in the presence of rotation and a mean magnetic field. Deissler $(1958,1960)$ developed a theory 'on the decay of homogeneous turbulence for times before the final period.' By considering Deissler's theory, Loeffler and Deissler (1961) studied the decay of temperature fluctuation in homogeneous turbulence before the final period. Bkar Pk et al. (2013) illustrated the decay of MHD turbulence prior to the ultimate phase in presence of dust particle for four-point correlation. Chandrasekhar (1951) obtained the invariant theory of isotropic turbulence in magneto-hydrodynamics. Rahaman (2010) obtained the decay of first order reactant in incompressible MHD turbulence before the final period for the case of multi-point and multi-time in a rotating system. Corrsin (1951) considered the spectrum of isotropic temperature fluctuations in isotropic turbulence. In their approach they considered the two- and three-point correlation equations and solved these equations after neglecting the fourth and higher order correlation terms analytically. Here, two- and three-point correlation equations have been considered, and the same approach of Deissler (1960) is applied to a theory of decaying homogeneous turblence. Sarker and Kishore (1991) derived the problem decay of the MHD turbulence before the final period analytically. In this chapter, we have discussed the problem numerically and represented the results graphically. We have shown that if the magnetic diffusivity is constant and kinematic viscosity is transferable then the fluctuation of the decay curves is linear 
and is parallel to the direction of $x$-axis and the decay is very small at constant time.

\section{Mathematical Formulation}

For two points, we need two equations. Let the induction equation of magnetic field at the point $P$ is

$$
\frac{\partial h_{i}}{\partial t}+u_{k} \frac{\partial h_{i}}{\partial x_{k}}-h_{k} \frac{\partial u_{i}}{\partial x_{k}}=\left(\frac{\vartheta}{P_{M}}\right) \frac{\partial^{2} h_{i}}{\partial x_{k} \partial x_{k}}
$$

and at the point $P^{\prime}$ will be

$$
\frac{\partial h_{j}{ }^{\prime}}{\partial t}+u_{k} \frac{\partial h_{j}{ }^{\prime}}{\partial x_{k}{ }^{\prime}}-h_{k} \frac{\partial u_{j}{ }^{\prime}}{\partial x_{k}{ }^{\prime}}=\left(\frac{\vartheta}{P_{M}}\right) \frac{\partial^{2} h_{j}{ }^{\prime}}{\partial x_{k}{ }^{\prime} \partial x_{k}{ }^{\prime}}
$$

where

$$
W=\frac{P}{\rho}+\frac{1}{2}|\vec{h}|^{2} \text { is the total MHD pressure, }
$$

$p(x, t)$ is the hydrodynamic pressure,

$\rho$ is the fluid density,

$P_{M}=\frac{\vartheta}{\lambda}$ is defined as the magnetic prandtle number,

$\vartheta$ is the kinematic viscosity,

$\lambda$ is the magnetic diffusivity,

$h_{i}(x, t)$ is the magnetic field fluctuation,

$u_{i}(x, t)$ is the turbulent velocity,

$t$ is the time, $x_{k}$ is the space coordinate, and the repeated subscripts are summed from 1 to 3 .

Multiplying the equation (1) by $h_{j}{ }^{\prime}$ and (2) by $h_{i}$ we get respectively

$$
\begin{gathered}
h_{j}{ }^{\prime} \frac{\partial h_{i}}{\partial t}+h_{j}{ }^{\prime} u_{k} \frac{\partial h_{i}}{\partial x_{k}}-h_{j}{ }^{\prime} h_{k} \frac{\partial u_{i}}{\partial x_{k}}=\left(\frac{\vartheta}{P_{M}}\right) h_{j}{ }^{\prime} \frac{\partial^{2} h_{i}}{\partial x_{k} \partial x_{k}} \\
h_{i} \frac{\partial h_{j}{ }^{\prime}}{\partial t}+h_{i} u_{k} \frac{\partial h_{j}{ }^{\prime}}{\partial x_{k}{ }^{\prime}}-h_{i} h_{k} \frac{\partial u_{j}{ }^{\prime}}{\partial x_{k}{ }^{\prime}}=\left(\frac{\vartheta}{P_{M}}\right) h_{i} \frac{\partial^{2} h_{j}{ }^{\prime}}{\partial x_{k}{ }^{\prime} \partial x_{k}{ }^{\prime}}
\end{gathered}
$$

Adding equation (3) and (4) and taking ensemble average with transformations

$$
\frac{\partial}{\partial r_{k}}=-\frac{\partial}{\partial x_{k}}=\frac{\partial}{\partial x_{k}{ }^{\prime}}
$$

and Chandrasekhar's relation (1951)

$$
\left\langle u_{k} h_{i} h_{j}{ }^{\prime}\right\rangle=-\left\langle h_{i} u_{k}{ }^{\prime} h_{j}{ }^{\prime}\right\rangle \text { and }\left\langle h_{i} u_{j}{ }^{\prime} h_{k}{ }^{\prime}\right\rangle=-\left\langle u_{i} h_{k} h_{j}{ }^{\prime}\right\rangle
$$

we obtain

$$
\begin{gathered}
\frac{\partial}{\partial t}\left\langle h_{i} h_{j}{ }^{\prime}\right\rangle+2\left[\frac{\partial}{\partial r_{k}}\left\langle u_{k}{ }^{\prime} h_{i} h_{j}{ }^{\prime}\right\rangle-\frac{\partial}{\partial r_{k}}\left\langle u_{i} h_{k} h_{j}{ }^{\prime}\right\rangle\right] \\
=2 \frac{\vartheta}{P_{M}} \frac{\partial^{2}}{\partial r_{k} \partial r_{k}}\left\langle h_{i} h_{j}{ }^{\prime}\right\rangle
\end{gathered}
$$

We have three dimensional Fourier transforms

$$
\begin{aligned}
& \left\langle h_{i} h_{j}{ }^{\prime}\right\rangle(\vec{r})=\int_{-\infty}^{\infty}\left\langle\Psi_{i} \Psi_{j}{ }^{\prime}\right\rangle(\vec{K}) \exp (\mathrm{i} \vec{K} \cdot \vec{r}) d \vec{K} \\
& \left\langle u_{i} h_{k} h_{j}{ }^{\prime}\right\rangle(\vec{r})=\int_{-\infty}^{\infty}\left\langle\alpha_{i} \Psi_{k} \Psi_{j}{ }^{\prime}\right\rangle(\vec{K}) \exp (\mathrm{i} \vec{K} \cdot \vec{r}) d \vec{K}
\end{aligned}
$$

Integrating the subscripts $i$ and $j$ and then integrating the points $P$ and $P^{\prime}$, we have

$$
\begin{aligned}
\left\langle u_{k} h_{i} h_{j}{ }^{\prime}\right\rangle(\vec{r}) & =\left\langle u_{k} h_{i} h_{j}{ }^{\prime}\right\rangle(-\vec{r}) \\
& =\int_{-\infty}^{\infty}\left\langle\alpha_{k} \Psi_{i} \Psi_{j}{ }^{\prime}\right\rangle(-\vec{K}) \exp (\mathrm{i} \vec{K} \cdot \vec{r}) d \vec{K}
\end{aligned}
$$

Putting these three equations in (5) becomes

$$
\begin{aligned}
& \frac{\partial}{\partial t}\left\langle\Psi_{i} \Psi_{j}{ }^{\prime}\right\rangle(\vec{K})+2 \frac{\vartheta}{P_{M}} k^{2}\left\langle\Psi_{i} \Psi_{j}{ }^{\prime}\right\rangle(\vec{K}) \\
= & 2 i k_{k}\left[\left\langle\alpha_{i} \Psi_{k} \Psi_{j}{ }^{\prime}\right\rangle(\vec{K})-\left\langle\alpha_{k} \Psi_{i} \Psi_{j}{ }^{\prime}\right\rangle(-\vec{K})\right]
\end{aligned}
$$

The above tensor equation becomes a scalar equation by contraction of the indices $i$ and $j$

$$
\begin{aligned}
& \frac{\partial}{\partial t}\left\langle\Psi_{i} \Psi_{i}{ }^{\prime}\right\rangle(\vec{K})+2 \frac{\vartheta}{P_{M}} k^{2}\left\langle\Psi_{i} \Psi_{i}{ }^{\prime}\right\rangle(\vec{K}) \\
& \quad=2 i k_{k}\left[\left\langle\alpha_{i} \Psi_{k} \Psi_{i}{ }^{\prime}\right\rangle(\vec{K})-\left\langle\alpha_{k} \Psi_{i} \Psi_{i}{ }^{\prime}\right\rangle(-\vec{K})\right]
\end{aligned}
$$

The term on the right hand side of the equation (9) is called energy transfer term while the second term on the left hand side is the dissipation term.

We consider the momentum equation of MHD turbulence at the point $P$, and the induction equations of magnetic field fluctuation at $P^{\prime}$ and $P^{\prime \prime}$ as follows

$$
\begin{aligned}
& \frac{\partial u_{l}}{\partial t}+u_{k} \frac{\partial u_{l}}{\partial x_{k}}-h_{k} \frac{\partial h_{l}}{\partial x_{k}}=-\frac{\partial W}{\partial x_{l}}+\vartheta \frac{\partial^{2} u_{l}}{\partial x_{k} \partial x_{k}} \\
& \frac{\partial h_{i}{ }^{\prime}}{\partial t}+u_{k}{ }^{\prime} \frac{\partial h_{i}{ }^{\prime}}{\partial x_{k}{ }^{\prime}}-h_{k}{ }^{\prime} \frac{\partial u_{i}{ }^{\prime}}{\partial x_{k}{ }^{\prime}}=\frac{\vartheta}{P_{M}} \frac{\partial^{2} h_{i}{ }^{\prime}}{\partial x_{k}{ }^{\prime} \partial x_{k}{ }^{\prime}} \\
& \frac{\partial h_{j}{ }^{\prime \prime}}{\partial t}+u_{k}{ }^{\prime \prime} \frac{\partial h_{j}{ }^{\prime \prime}}{\partial x_{k}{ }^{\prime \prime}}-h_{k}{ }^{\prime \prime} \frac{\partial u_{j}{ }^{\prime \prime}}{\partial x_{k}{ }^{\prime \prime}}=\frac{\vartheta}{P_{M}} \frac{\partial^{2} h_{j}{ }^{\prime \prime}}{\partial x_{k}{ }^{\prime \prime} \partial x_{k}{ }^{\prime \prime}}
\end{aligned}
$$

Multiplying equation (10) by $h_{i} h_{j}{ }^{\prime \prime}$, (11) by $u_{i} h_{j}{ }^{\prime \prime}$ and (12) by $u_{i} h_{i}{ }^{\prime}$, we obtain

$$
\begin{gathered}
h_{i} h_{j}{ }^{\prime \prime} \frac{\partial u_{l}}{\partial t}+u_{k} h_{i} h_{j}{ }^{\prime \prime} \frac{\partial u_{l}}{\partial x_{k}}-h_{k} h_{i} h_{j}{ }^{\prime \prime} \frac{\partial h_{l}}{\partial x_{k}} \\
=-h_{i} h_{j}{ }^{\prime \prime} \frac{\partial W}{\partial x_{l}}+\vartheta h_{i} h_{j}{ }^{\prime \prime} \frac{\partial^{2} u_{l}}{\partial x_{k} \partial x_{k}} \\
u_{i} h_{j}{ }^{\prime \prime} \frac{\partial h_{i}{ }^{\prime}}{\partial t}+u_{k}{ }^{\prime} u_{i} h_{j}{ }^{\prime \prime} \frac{\partial h_{i}{ }^{\prime}}{\partial x_{k}{ }^{\prime}}-h_{k}{ }^{\prime} u_{i} h_{j}{ }^{\prime \prime} \frac{\partial u_{i}{ }^{\prime}}{\partial x_{k}{ }^{\prime}} \\
=\frac{\vartheta}{P_{M}} u_{i} h_{j}{ }^{\prime \prime} \frac{\partial^{2} h_{i}{ }^{\prime}}{\partial x_{k}{ }^{\prime} \partial x_{k}{ }^{\prime}} \\
u_{i} h_{i}{ }^{\prime} \frac{\partial h_{j}{ }^{\prime \prime}}{\partial t}+u_{k}{ }^{\prime \prime} u_{i} h_{i}{ }^{\prime} \frac{\partial h_{j}{ }^{\prime \prime}}{\partial x_{k}{ }^{\prime \prime}}-h_{k}{ }^{\prime \prime} u_{i} h_{i}{ }^{\prime} \frac{\partial u_{j}{ }^{\prime \prime}}{\partial x_{k}{ }^{\prime \prime}} \\
=\frac{\vartheta}{P_{M}} u_{i} h_{i}{ }^{\prime} \frac{\partial^{2} h_{j}{ }^{\prime \prime}}{\partial x_{k}{ }^{\prime \prime} \partial x_{k}{ }^{\prime \prime}}
\end{gathered}
$$

Combining equations (13), (14), (15), and taking time average and using the transformation, we obtain

$$
\frac{\partial}{\partial x_{k}{ }^{\prime}}=\frac{\partial}{\partial r_{k}}, \frac{\partial}{\partial x_{k}{ }^{\prime \prime}}=\frac{\partial}{\partial r_{k}{ }^{\prime}},
$$

and 


$$
\frac{\partial}{\partial x_{k}}=-\left(\frac{\partial}{\partial r_{k}}+\frac{\partial}{\partial r_{k}{ }^{\prime}}\right)
$$

We get

$$
\begin{array}{r}
\frac{\partial}{\partial t}\left\langle u_{l} h_{i}{ }^{\prime}, h_{j}{ }^{\prime \prime}\right\rangle-\frac{\vartheta}{P_{M}}\left[\left(1+P_{M}\right) \frac{\partial^{2}}{\partial r_{k} \partial r_{k}}\left\langle u_{l} h_{i}{ }^{\prime} h_{j}{ }^{\prime \prime}\right\rangle+\right. \\
\left.\left(1+P_{M}\right) \frac{\partial^{2}}{\partial r_{k}{ }^{\prime} \partial r_{k}{ }^{\prime}}\left\langle u_{l} h_{i}{ }^{\prime} h_{j}{ }^{\prime \prime}\right\rangle+2 P_{M} \frac{\partial^{2}}{\partial r_{k} \partial r_{k}{ }^{\prime}}\left\langle u_{l} h_{i}{ }^{\prime} h_{j}{ }^{\prime \prime}\right\rangle\right]= \\
\frac{\partial}{\partial r_{k}}\left\langle u_{l} u_{k} h_{i}{ }^{\prime} h_{j}{ }^{\prime \prime}\right\rangle+\frac{\partial}{\partial r_{k}{ }^{\prime}}\left\langle u_{l} u_{k} h_{i}{ }^{\prime} h_{j}{ }^{\prime \prime}\right\rangle-\frac{\partial}{\partial r_{k}{ }^{\prime}}\left\langle h_{l} h_{k} h_{i}{ }^{\prime} h_{j}{ }^{\prime \prime}\right\rangle- \\
\frac{\partial}{\partial r_{k}{ }^{\prime}}\left\langle h_{l} h_{k} h_{i}{ }^{\prime} h_{j}{ }^{\prime \prime}\right\rangle-\frac{\partial}{\partial r_{k}}\left\langle u_{l} u_{k}{ }^{\prime} h_{i}{ }^{\prime} h_{j}{ }^{\prime \prime}\right\rangle+\frac{\partial}{\partial r_{k}}\left\langle u_{l} u_{i}{ }^{\prime} h_{k}{ }^{\prime} h_{j}{ }^{\prime \prime}\right\rangle- \\
\frac{\partial}{\partial r_{k}{ }^{\prime}}\left\langle u_{l} u_{k}{ }^{\prime \prime} h_{i}{ }^{\prime} h_{j}{ }^{\prime \prime}\right\rangle+\frac{\partial}{\partial r_{k}{ }^{\prime}}\left\langle u_{l} u_{j}{ }^{\prime \prime} h_{i}{ }^{\prime} h_{k}{ }^{\prime \prime}\right\rangle+\frac{\partial}{\partial r_{l}}\left\langle\left(W h_{i}{ }^{\prime} h_{j}{ }^{\prime \prime}\right\rangle+\right. \\
\frac{\partial}{\partial r_{l}{ }^{\prime}}\left\langle W h_{i}{ }^{\prime} h_{j}{ }^{\prime \prime}\right\rangle
\end{array}
$$

Using Fourier transforms

$$
\left\langle u_{l} h_{i}{ }^{\prime}(\vec{r}), h_{j}{ }^{\prime \prime}\left(\vec{r}^{\prime}\right)\right\rangle=
$$$$
\int_{-\infty}^{\infty} \int_{-\infty}^{\infty}\left\langle\Phi_{1} \beta_{i}{ }^{\prime}(\vec{K}) \beta_{j}{ }^{\prime \prime}\left(\vec{K}^{\prime}\right)\right\rangle \cdot \exp \left[\mathrm{i}\left(\vec{K} \cdot \vec{r}+\vec{K}^{\prime} \cdot \vec{r}^{\prime}\right)\right] d \vec{K} d \vec{K}^{\prime}
$$

and

$$
\begin{gathered}
\left\langle W h_{i}{ }^{\prime}(\vec{r}), h_{j}{ }^{\prime \prime}\left(\vec{r}^{\prime}\right)\right\rangle= \\
\int_{-\infty}^{\infty} \int_{-\infty}^{\infty}\left\langle\gamma \beta_{i}{ }^{\prime}(\vec{K}) \beta_{j}{ }^{\prime \prime}\left(\vec{K}^{\prime}\right)\right\rangle \cdot \exp \left[\mathrm{i}\left(\vec{K} \cdot \vec{r}+\vec{K}^{\prime} \cdot \vec{r}^{\prime}\right)\right] d \vec{K} d \vec{K}^{\prime}
\end{gathered}
$$

with transformation

$$
\left\langle u_{l} u_{k}{ }^{\prime \prime} h_{j}{ }^{\prime \prime} h_{i}{ }^{\prime}\right\rangle=\left\langle u_{l} u_{k}{ }^{\prime} h_{i}{ }^{\prime} h_{j}{ }^{\prime \prime}\right\rangle
$$

and

$$
\left\langle u_{l} u_{j}{ }^{\prime \prime} h_{i}{ }^{\prime} h_{k}{ }^{\prime \prime}\right\rangle=\left\langle u_{l} u_{i}{ }^{\prime} h_{k}{ }^{\prime} h_{j}{ }^{\prime \prime}\right\rangle
$$

The equation (17) reduces into the form

$$
\begin{array}{r}
\frac{\partial}{\partial t}\left\langle\Phi_{l} \beta_{i}{ }^{\prime} \beta_{j}{ }^{\prime \prime}\right\rangle+\frac{\vartheta}{P_{M}}\left[\left(1+P_{M}\right) k^{2}+\left(1+P_{M}\right) k^{\prime 2}+\right. \\
\left.2 P_{M} k_{k} k_{k}{ }^{\prime}\right]\left\langle\Phi_{l} \beta_{i}{ }^{\prime \prime} \beta_{j}{ }^{\prime \prime}\right\rangle=i\left(k_{k}+k_{k}{ }^{\prime}\right)\left\langle\Phi_{l} \Phi_{k} \beta_{i}{ }^{\prime} \beta_{j}{ }^{\prime \prime}\right\rangle- \\
i\left(k_{k}+k_{k}{ }^{\prime}\right)\left\langle\beta_{l} \beta_{k} \beta_{i}{ }^{\prime \prime} \beta_{j}{ }^{\prime \prime}-i\left(k_{k}+k_{k}{ }^{\prime}\right)\left\langle\Phi_{l} \Phi_{k}{ }^{\prime} \beta_{i}{ }^{\prime \prime} \beta_{j}\right\rangle+\right. \\
i\left(k_{k}+k_{k}{ }^{\prime}\right)\left\langle\Phi_{l} \Phi_{i}{ }^{\prime} \beta_{k}{ }^{\prime} \beta_{j}{ }^{\prime \prime}\right\rangle+i\left(k_{l}+k_{l}{ }^{\prime}\right)\left\langle\gamma \beta_{i}{ }^{\prime} \beta_{j}{ }^{\prime \prime}\right\rangle
\end{array}
$$

Taking contraction of the indices $i$ and $j$ in equation (19), we get the spectrum equation at three point correlation as:

$$
\begin{aligned}
\frac{\partial}{\partial t}\left\langle\Phi_{l} \beta_{i}{ }^{\prime} \beta_{i}{ }^{\prime \prime}\right\rangle+\frac{\vartheta}{P_{M}} & {\left[\left(1+P_{M}\right)\left(k^{2}+k^{\prime 2}\right)\right.} \\
& \left.+2 P_{M} k k^{\prime}\right]\left\langle\Phi_{l} \beta_{i}{ }^{\prime} \beta_{i}{ }^{\prime \prime}\right\rangle \\
& =i\left(k_{k}+k_{k}{ }^{\prime}\right)\left\langle\Phi_{l} \Phi_{k} \beta_{i}^{\prime} \beta_{i}^{\prime \prime}\right\rangle \\
& -i\left(k_{k}+k_{k}{ }^{\prime}\right)\left\langle\beta_{l} \beta_{k} \beta_{i}^{\prime} \beta_{i}^{\prime \prime}\right\rangle \\
& -i\left(k_{k}+k_{k}{ }^{\prime}\right)\left\langle\Phi_{l} \Phi_{k} \beta_{i}^{\prime} \beta_{i}^{\prime \prime}\right\rangle+
\end{aligned}
$$

$$
i\left(k_{k}+k_{k}{ }^{\prime}\right)\left\langle\Phi_{l} \Phi_{i}{ }^{\prime} \beta_{k}{ }^{\prime} \beta_{i}{ }^{\prime \prime}\right\rangle+i\left(k_{l}+k_{l}{ }^{\prime}\right)\left\langle\gamma \beta_{i}{ }^{\prime} \beta_{i}{ }^{\prime \prime}\right\rangle
$$

Taking derivative of equation (10) at $P$ with respect to $x_{l}$ we get

$$
-\frac{\partial^{2} \mathrm{~W}}{\partial x_{l} \partial x_{l}}=\frac{\partial^{2}}{\partial x_{l} \partial x_{k}}\left(u_{l} u_{k}-h_{l} h_{k}\right)
$$

For independent variables $r$ and $r^{\prime}$ multiplying equation (21) by $h_{i}{ }^{\prime} h_{j}{ }^{\prime \prime}$, taking time averages we get

$$
\begin{gathered}
-\left[\frac{\partial^{2}}{\partial r_{l} \partial r_{l}}+\frac{\partial^{2}}{\partial r_{l}{ }^{\prime} \partial r_{l}{ }^{\prime}}+2 \frac{\partial^{2}}{\partial r_{l} \partial r_{l}{ }^{\prime}}\right]\left\langle W h_{i}{ }^{\prime} h_{j}{ }^{\prime \prime}\right\rangle=\left[\frac{\partial^{2}}{\partial r_{l} \partial r_{k}}+\frac{\partial^{2}}{\partial r_{l}{ }^{\prime} \partial r_{k}}+\right. \\
\left.\frac{\partial^{2}}{\partial r_{l} \partial r_{k}}+\frac{\partial^{2}}{\partial r_{l}{ }^{\prime} \partial r_{k}{ }^{\prime}}\right] \cdot\left(\left\langle u_{l} u_{k} h_{i}{ }^{\prime} h_{j}{ }^{\prime \prime}\right\rangle-\left\langle h_{l} h_{k} h_{i}{ }^{\prime} h_{j}{ }^{\prime}\right\rangle\right)
\end{gathered}
$$

Applying Fourier transforms of the equation (22), we get

$-\left\langle\gamma \beta_{i}{ }^{\prime} \beta_{j}{ }^{\prime \prime}\right\rangle=\frac{\left(k_{l} k_{k}+k_{l}{ }^{\prime} k_{k}+k_{l} k_{k}{ }^{\prime}\right)\left(\left\langle\Phi_{l} \Phi_{k} \beta_{i}{ }^{\prime} \beta_{j}{ }^{\prime \prime}\right\rangle-\left\langle\beta_{l} \beta_{k} \beta_{i}{ }^{\prime} \beta_{j}{ }^{\prime \prime}\right\rangle\right)}{k^{2}+k^{\prime 2}+2 k_{l} k_{l}{ }^{\prime}}$

where $\left\langle\gamma \beta_{i}{ }^{\prime} \beta_{j}{ }^{\prime \prime}\right\rangle$ is the pressure correlation.

\section{Results and Discussion}

The solution is obtained by considering the two-point correlation after neglecting the third order correlations the three point correlation equations is considered and the quadruple correlations are neglected. The terms $\left\langle\gamma \beta_{i}{ }^{\prime} \beta_{j}{ }^{\prime \prime}\right\rangle$ associated with the pressure correlations must be neglected. Thus, neglecting all the terms on the right hand side of the equation (2), we have

$$
\begin{gathered}
\frac{\partial}{\partial t}\left\langle\Phi_{l} \beta_{i}{ }^{\prime} \beta_{j}{ }^{\prime \prime}\right\rangle+ \\
\frac{\vartheta}{P_{M}}\left[\left(1+P_{M}\right)\left(K^{2}+K^{\prime 2}\right)+2 P_{M} K K^{\prime}\right]\left\langle\Phi_{l} \beta_{i}{ }^{\prime} \beta_{j}{ }^{\prime \prime}\right\rangle=0
\end{gathered}
$$

Integrating the equation (24) between $t_{0}$ and $t$ with inner multiplication by $k_{k}$ and gives

$$
\begin{array}{r}
k_{k}\left\langle\Phi_{l} \beta_{i}{ }^{\prime} \beta_{j}{ }^{\prime \prime}\right\rangle=k_{k}\left\langle\Phi_{l} \beta_{i}{ }^{\prime} \beta_{j}{ }^{\prime \prime}\right\rangle_{0} \exp \left[-\frac{\vartheta}{P_{M}}\left\{\left(1+P_{M}\right)\left(k^{2}+{k^{\prime}}^{2}\right)+\right.\right. \\
\left.\left.2 P_{M} K K^{\prime} \cos \theta\right\}\left(t_{0}-t\right)\right]
\end{array}
$$

Where $\theta$ is the angle between $k$ and $k^{\prime}$.

Now, by letting $r^{\prime}=0$ in the equation (18) and comparing with the equation (7) and (8), we obtain

$$
\begin{gathered}
\left\langle\alpha_{i} \Psi_{k} \Psi_{i}{ }^{\prime}\right\rangle(\vec{K})=\int_{-\infty}^{\infty}\left\langle\Phi_{l} \beta_{i}{ }^{\prime} \beta_{i}{ }^{\prime \prime}\right\rangle \mathrm{d} \vec{K} \\
\left\langle\alpha_{k} \Psi_{i} \Psi_{i}{ }^{\prime}\right\rangle(-\vec{K})=\int_{-\infty}^{\infty}\left\langle\Phi_{l} \beta_{i}{ }^{\prime}(-\vec{K}) \beta_{i}{ }^{\prime \prime}\left(-\vec{K}^{\prime}\right)\right\rangle \mathrm{d} \vec{K}
\end{gathered}
$$

Substituting the equations (25), (26) and (27) in equation (9), we get

$$
\begin{gathered}
\frac{\partial}{\partial t}\left\langle\Psi_{i} \Psi_{i}{ }^{\prime}\right\rangle(\vec{K})+2 \frac{\vartheta}{P_{M}} K^{2}\left\langle\Psi_{i} \Psi_{i}{ }^{\prime}\right\rangle(\vec{K}) \\
=2 i k_{k}\left[\left\langle\Phi_{l} \beta_{i}{ }^{\prime} \beta_{i}{ }^{\prime \prime}\right\rangle-\left\langle\Phi_{l} \beta_{i}{ }^{\prime}(-\vec{K}) \beta_{i}{ }^{\prime \prime}\left(-\vec{K}^{\prime}\right)\right\rangle\right]_{0} \\
\cdot \exp \left[-\frac{\vartheta}{P_{M}}\left\{\left(1+P_{M}\right)\left(k^{2}+{k^{\prime}}^{2}\right)+2 P_{M} k k^{\prime} \cos \theta\right\}\left(t-t_{0}\right)\right] \mathrm{d} \vec{K}^{\prime}
\end{gathered}
$$

Now, $\mathrm{d} \vec{K}^{\prime}=\mathrm{d} K_{1}^{\prime} \mathrm{d} K_{2}^{\prime} \mathrm{d} K_{3}^{\prime}$ can be expressed in terms of $k^{\prime}$ and $\theta$.

Following Sarker and kishore (1991) and using Loeffler and Deissler (1961) and assumption and then integrating 
w.r.to $\cos \theta$, we get,

$$
\frac{\partial H}{\partial t}+\frac{2 \vartheta k^{2} H}{P_{M}}=G
$$

where $H=2 \pi k^{2}\left\langle\Psi_{i} \Psi_{i}{ }^{\prime}\right\rangle(\vec{K})$ is the magnetic energy spectrum function and $G$ is the energy transfer term given by

$$
\begin{array}{r}
G=-\frac{\xi_{0}}{\vartheta\left(t-t_{0}\right)} \int_{0}^{\infty}\left(k^{3} k^{\prime 5}-k^{5} k^{\prime 3}\right) \cdot\left[\exp \left\{-\frac{\vartheta}{P_{M}}\left(t-t_{0}\right)\left[\left(1+P_{M}\right)\left(k^{2}+k^{\prime 2}\right)-2 P_{M} k k^{\prime}\right]\right\}-\exp \left\{-\frac{\vartheta}{P_{M}}\left(t-t_{0}\right)\left[( 1 + P _ { M } ) \left(k^{2}+\right.\right.\right.\right. \\
\left.\left.\left.\left.k^{\prime 2}\right)+2 P_{M} k k^{\prime}\right]\right\}\right] d k^{\prime}
\end{array}
$$

Integrating equation (30) with respect to $k^{\prime}$, we have

$$
G=-\frac{-\sqrt{\pi} \xi_{0} P_{M}^{\frac{5}{2}}}{\vartheta^{\frac{3}{2}}\left(t-t_{0}\right)^{\frac{3}{2}}\left(1+P_{M}\right)^{\frac{5}{2}}} \exp \left\{\frac{-\vartheta\left(t-t_{0}\right)}{P_{M}} \cdot\left(\frac{1+2 P_{M}}{1+P_{M}}\right) k^{2}\right\}\left[\frac{15 P_{M} k^{4}}{4 \vartheta^{2}\left(t-t_{0}\right)^{2}\left(1+P_{M}\right)}+\left\{\frac{5 P_{M}^{2}}{\left(1+P_{M}\right)^{2} \vartheta\left(t-t_{0}\right)}-\frac{3}{2 \vartheta\left(t-t_{0}\right)}\right\} k^{6}+\frac{P_{M}}{1+P_{M}}\left\{\frac{P_{M}{ }^{2}}{\left(1+P_{M}\right)^{2}}-1\right\} k^{8}\right]
$$

Solving the linear equation (29)and using Corrsin(1951) relation $J(k)=\frac{N_{0} k^{2}}{\pi}$ is a constant, we get

$$
H=\frac{N_{0} k^{2}}{\pi} \exp \left[-2 \frac{\vartheta k^{2}\left(t-t_{0}\right)}{P_{M}}\right]+\frac{\sqrt{\pi} \xi_{0} P_{M}^{\frac{5}{2}}}{\vartheta^{\frac{3}{2}}\left(1+P_{M}\right)^{\frac{7}{2}}} \cdot \exp \left\{\frac{-\vartheta k^{2}\left(t-t_{0}\right)\left(1+2 P_{M}\right)}{P_{M}\left(1+P_{M}\right)}\right\}\left[\frac{3 P_{M} k^{4}}{2 \vartheta^{2}\left(t-t_{0}\right)^{\frac{5}{2}}}+\frac{\left(7 P_{M}^{2}-6 P_{M}\right) k^{6}}{3\left(1+P_{M}\right)\left(t-t_{0}\right)^{\frac{3}{2}}}-\frac{4\left(3 P_{M}^{2}-2 P_{M}+3\right) k^{8}}{3\left(1+P_{M}\right)^{2}\left(t-t_{0}\right)^{\frac{1}{2}}}+\right.
$$

where

$$
F(\omega)=e^{-\omega^{2}} \int_{0}^{\omega} e^{x^{2}} d x, \omega=k \sqrt{\left[\frac{\vartheta\left(t-t_{0}\right)}{P_{M}\left(1+P_{M}\right)}\right]}
$$

By setting

$$
\vec{r}=0, j=i
$$

$$
d \vec{K}=-2 \pi k^{2} d(\cos \theta) d k a n d H=2 \pi k^{2}\left\langle\Psi_{i} \Psi_{i}\right\rangle
$$

in the equation (20), we get the expression for magnetic energy decay as

$$
\frac{\left\langle\mathrm{h}_{i} \mathrm{~h}_{i}{ }^{\prime}\right\rangle}{2}=\int_{0}^{\infty} H d k
$$

Substituting equation (32) into (33) and after integration w. r.to time, we can write

where

$$
\left\langle h^{2}\right\rangle=A\left(t-t_{0}\right)^{-\frac{3}{2}}+B\left(t-t_{0}\right)^{-5}
$$

Table 1. If $\lambda$ is fixed and $\vartheta$ varies from 0.05 to 0.15 .

\begin{tabular}{llllll}
\hline $\boldsymbol{\vartheta}$ & $\boldsymbol{\lambda}$ & $\boldsymbol{P}_{\boldsymbol{M}}$ & $\boldsymbol{Q}$ & $\boldsymbol{A}$ & $\boldsymbol{B}$ \\
\hline 0.05 & 2 & 0.025 & $-4.96 \times 10^{-10}$ & $6.97 \times 10^{-5}$ & -0.064 \\
0.10 & 2 & 0.050 & $-7.24 \times 10^{-8}$ & $1.971 \times 10^{-4}$ & -0.144 \\
0.15 & 2 & 0.075 & $-1.08 \times 10^{-6}$ & $3.622 \times 10^{-4}$ & -0.190 \\
\hline
\end{tabular}

Table 2. Different values of $A$ and $B$ if $\lambda$ is constant.

\begin{tabular}{llllll}
\hline $\boldsymbol{\vartheta}$ & $\boldsymbol{\lambda}$ & $\boldsymbol{P}_{\boldsymbol{M}}$ & $\boldsymbol{Q}$ & $\boldsymbol{A}$ & $\boldsymbol{B}$ \\
\hline 0.20 & 2 & 0.100 & $-5.27 \times 10^{-6}$ & $5.576 \times 10^{-4}$ & -0.164 \\
0.25 & 2 & 0.125 & $-2.08 \times 10^{-6}$ & $7.791 \times 10^{-4}$ & -0.170 \\
0.30 & 2 & 0.150 & $-5.645 \times 10^{-5}$ & $1.024 \times 10^{-3}$ & -0.155 \\
\hline
\end{tabular}

Table 3. Values of $A$ and $B$ for the variation of $\vartheta$.

\begin{tabular}{llllll}
\hline $\boldsymbol{\vartheta}$ & $\boldsymbol{\lambda}$ & $\boldsymbol{P}_{\boldsymbol{M}}$ & $\boldsymbol{Q}$ & $\boldsymbol{A}$ & $\boldsymbol{B}$ \\
\hline 0.35 & 2 & 0.175 & $-1.262 \times 10^{-4}$ & $1.291 \times 10^{-3}$ & -0.137 \\
0.40 & 2 & 0.200 & $-2.453 \times 10^{-4}$ & $1.577 \times 10^{-3}$ & -0.120 \\
0.45 & 2 & 0.225 & $-4.293 \times 10^{-4}$ & $1.882 \times 10^{-3}$ & -0.103 \\
\hline
\end{tabular}

$$
\begin{gathered}
Q=\frac{\pi P_{M}^{6}}{\left(1+P_{M}\right)\left(1+2 P_{M}\right)^{\frac{5}{2}}}\left[\frac{9}{16}+\frac{5 P_{M}\left(7 P_{M}-6\right)}{\left(1+2 P_{M}\right)}\right. \\
\left.-\frac{35 P_{M}\left(3 P_{M}^{2}-2 P_{M}+3\right)}{8\left(1+2 P_{M}\right)^{2}}+\cdots\right] \\
A=\frac{N_{0} P_{M}^{\frac{3}{2}} \vartheta^{-\frac{3}{2}}}{8 \sqrt{2 \pi}} \\
B=\xi_{0} 2 Q \vartheta^{-6}
\end{gathered}
$$

which is the decay law for magnetic energy fluctuation before the final period.

Now we are going to discuss the problem in numerical analysis:

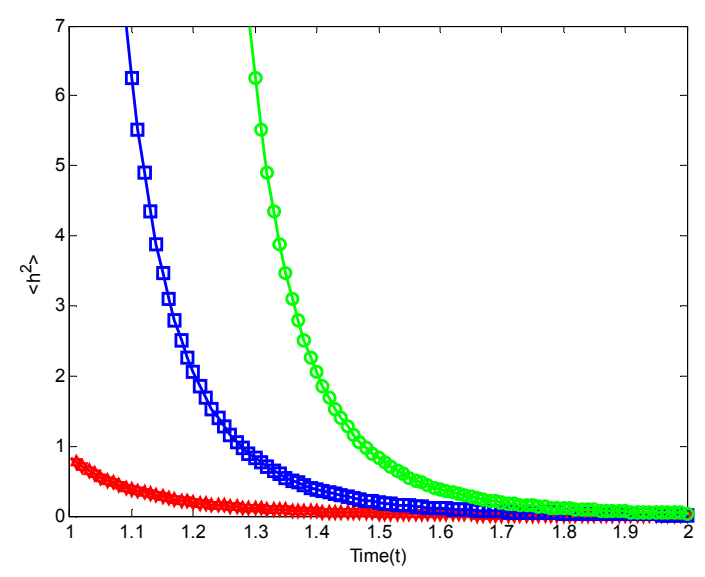

Figure 1. Energy decay curves for $A=6.97 \times 10^{-5}$ and $B=0.064$, $t_{0}=0.4,0.7$, and 0.9 . 


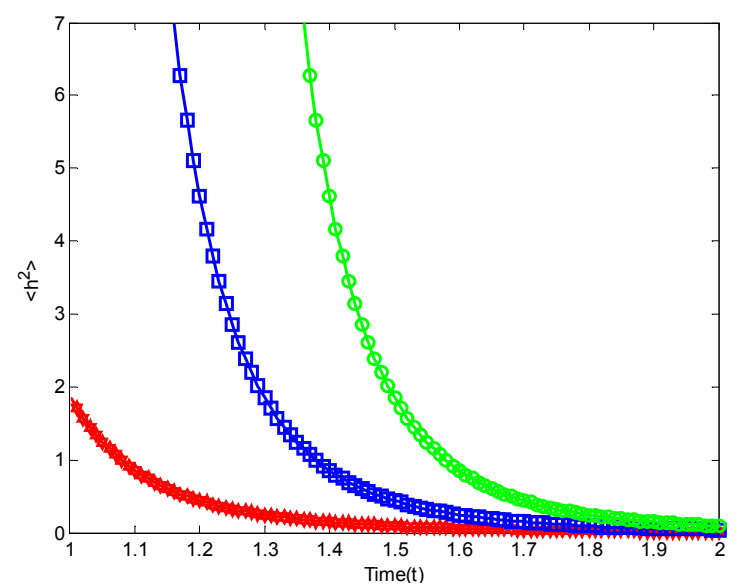

Figure 2. Energy decay curves for $A=1.971 \times 10^{-4}$ and $B=-0.144$, $t_{0}=0.4,0.7$, and 0.9 .

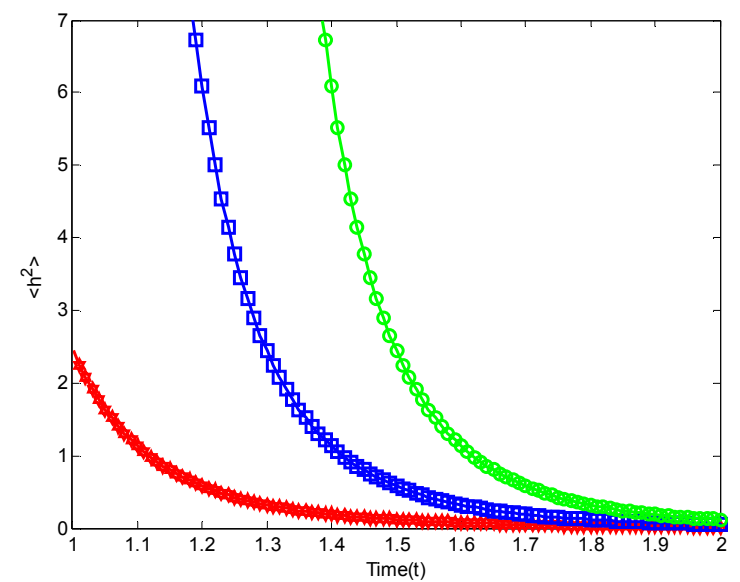

Figure 3. Energy decay curves for $A=3.622 \times 10^{-4}$ and $B=$ $-0.190, t_{0}=0.4,0.7$, and 0.9 .

If $\lambda$ (magnetic diffusivity) is fixed and $\vartheta$ (kinematic viscosity) varies from 0.05 to 0.15 in Table 1 , Magnetic Prandtle number $P_{M}$ is increased for increasing of kinematic viscosity $(\vartheta)$ because they are proportional to each other.

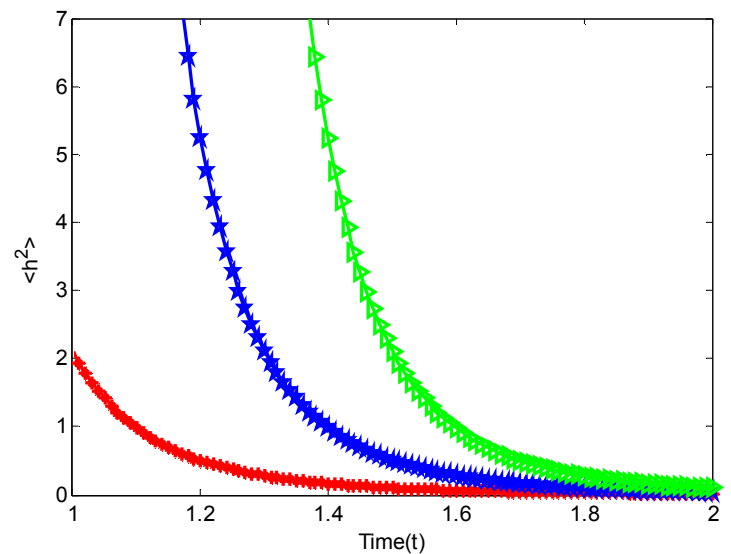

Figure 4. Energy decay curves for $A=5.576 \times 10^{-4}$ and $B=-0.164$, $t_{0}=0.4,0.7$, and 0.9 .

For fixed time $\left(t_{0}=0.4,0.7,0.9\right)$ and for different values of $A$ and $B$, the total energy $\left\langle h^{2}\right\rangle$ is increasing from Figure 1 to Figure 3. Here time $(t)$ has been taken in the direction of $x$-axis and total energy $\left\langle h^{2}\right\rangle$ in the direction of $y$-axis. In the direction of $x$-axis for time the limit has been taken from 1 to 2 and in the direction of $y$-axis for total energy the limit has been taken from 0 to 7 .

When $\lambda$ is fixed and $\vartheta$ varies from 0.20 to 0.30 , different values of A \& B in Table 2, the change of total energy is very small from Figure 4 to Figure 6.

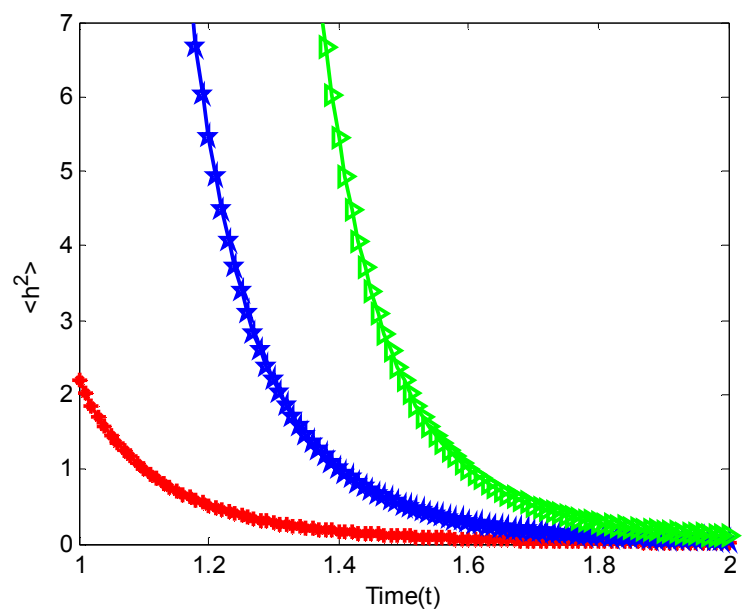

Figure 5. Energy decay curves for $A=7.791 \times 10^{-4}$ and $B=-0.170$, $t_{0}=0.4,0.7$, and 0.9 .

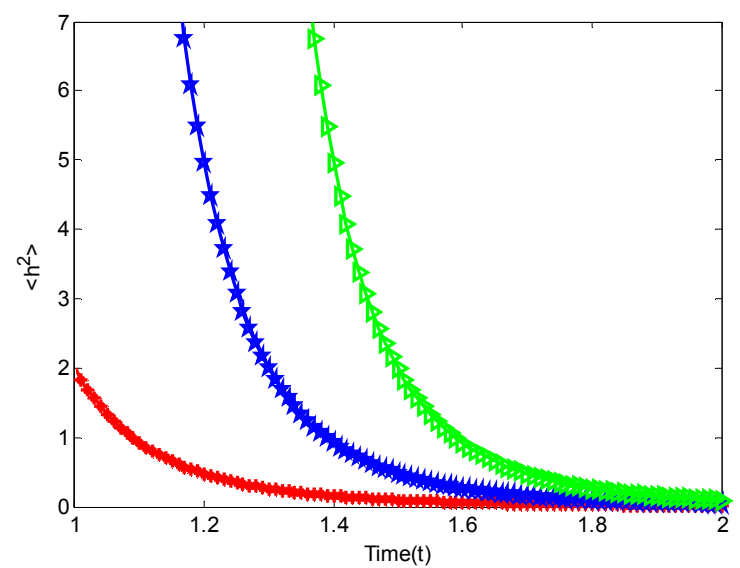

Figure 6. Energy decay curves for $A=1.024 \times 10^{-3}$ and $B=-0.155$, $t_{0}=0.4,0.7$, and 0.9 .

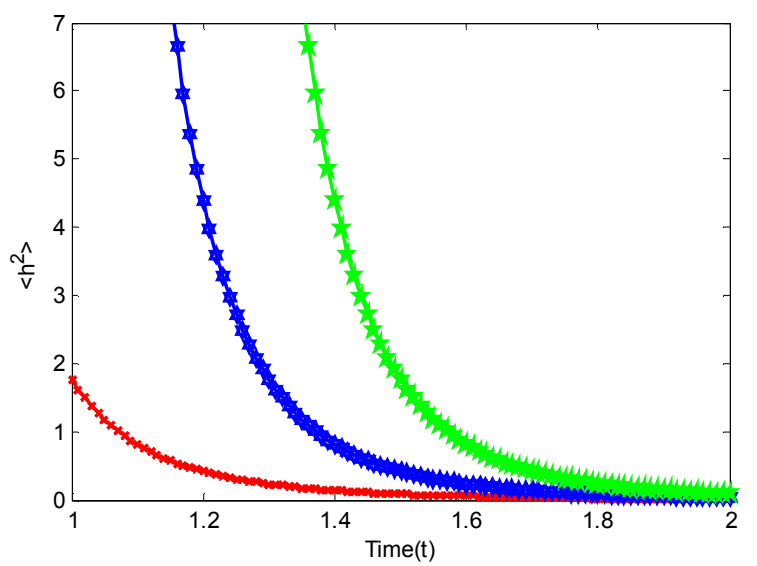

Figure 7. Energy decay curves for $A=1.291 \times 10^{-3}$ and $B=-0.137$, $t_{0}=0.4,0.7$, and 0.9 . 


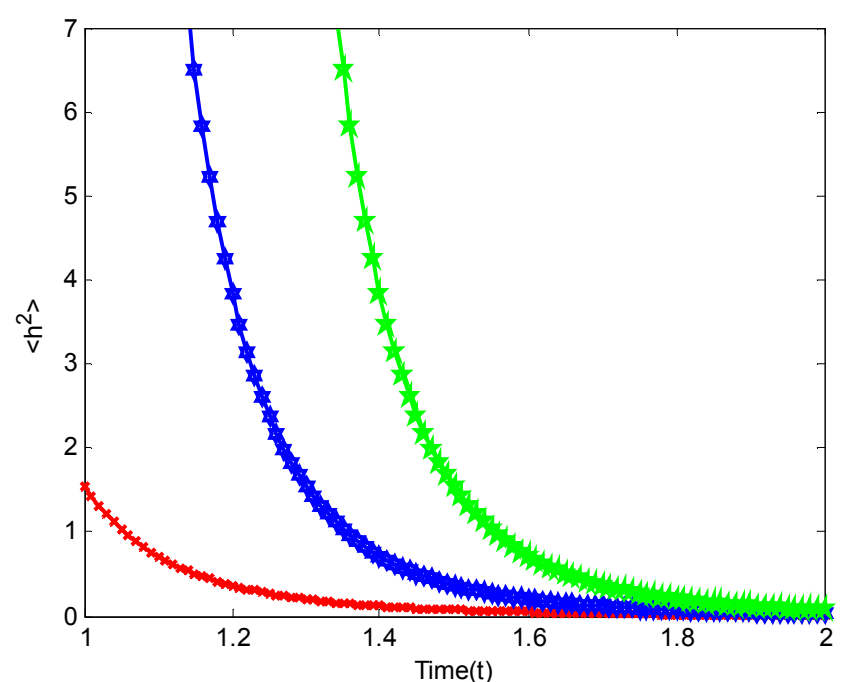

Figure 8. Energy decay curves for $A=1.577 \times 10^{-3}$ and $B=-0.120$, $t_{0}=0.4,0.7$, and 0.9 .

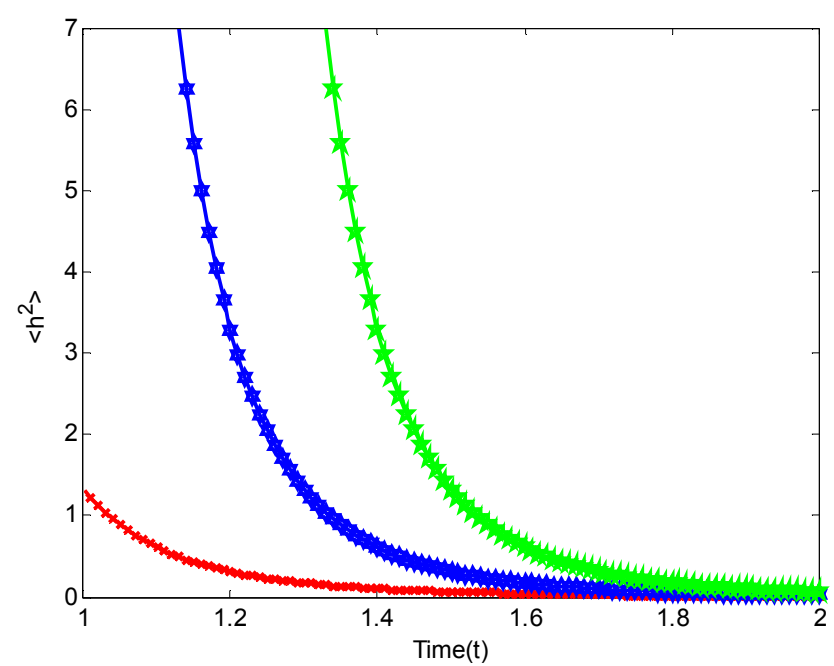

Figure 9. Energy decay curves for $A=1.882 \times 10^{-3}$ and $B=-0.103$, $t_{0}=0.4,0.7$, and 0.9 .

Similarly, if $\lambda$ is fixed and $\vartheta$ varies from 0.35 to 0.45 in Table 3, the straight line is smaller than that of Figure 5 which has been shown in the Figure 9.

But for fixed time and different values of $A \& B$ which has been indicated by Table 3 , the total energy is decreasing slowly from Figure 7 to Figure 9.

\section{Conclusion}

From the above tables, figures and discussion we conclude that the following results:

- When magnetic diffusivity is constant and kinematic viscosity is changeable then the Magnetic Prandtle number is proportional to the kinematic viscosity.

- In the absence of non-dimensional quantity the total energy of magnetic field fluctuation is decaying very rapidly.

- The magnetic field fluctuation of total energy is gradually increased at fixed time $t_{0}=0.4,0.7$, and 0.9 .
- The energy decay is very small at constant time which has been shown in the Figure 6 to Figure 8.

\section{Acknowledgements}

Authors (Ripan Roy \& M. Abu Bkar Pk) are grateful to the Department of Applied Mathematics, University of Rajshahi, Bangladesh for giving all facilities and support to carry out this work.

\section{References}

[1] Biskamp, D., "Magnetohydrodynamic turbulence." Cambridge U. P., UK, pp. 75-77, 2003.

[2] Bkar Pk, M. A. Abdul Malek, M. A. K. Azad, "Effects of First-Order Reactant on MHD Turbulence at Four-Point Correlation." Applied and Computational Mathematics, Vol. 4, Issue 1, pp. 11-19, 2015.

[3] Bkar Pk., M. A., M. S. A. Sarker and M. A. K. Azad, "Decay of MHD turbulence prior to the ultimate phase in presence of dust particle for four-point correlation." International Journal of Applied Mathematics and Mechanics, Vol. 9(10), pp.34-57, 2013.

[4] Boyd, J. P., "Chebyshev and Forier spectral method." second edition, Dover, Mineola, NY, 2001.

[5] Chandrasekhar, S., "The invariant theory of isotropic turbulence in magneto-hydrodynamics." Proc. Roy. Soc. London, A 204, 435, 1951.

[6] Corrsin, S., "The spectrum of isotropic temperature fluctuations in isotropic turbulence." Journal of Applied Physics, Vol. 22, pp. 469-473, 1951.

[7] Deissler, R. G., "A theory of decaying homogeneous turbulence." Physics of Fluids, Vol. 3, pp. 176-187, 1960.

[8] Deissler, R. G., "On the decay of homogeneous turbulence for times before the final period." Physics of Fluids, Vol. 1, pp. 111-121, 1958.

[9] Islam, M. A. and M. S. A. Sarker, "The first order reactant in MHD turbulence before the final period of decay for the case of multi-point and multi-time." Indian Journal of Pure and Applied Mathematics, Vol. 32, pp. 1173-1184, 2001.

[10] Loeffler, A. L. and R. G. Deissler, "Decay of temperature fluctuations inhomogeneous turbulence before the final period." International Journal of Heat Mass Transfer, Vol. 1, 312-324, 1961.

[11] Monuar Hossain, M., M. Abu Bkar Pk, M.S. Alam Sarker, "Homogeneous Fluid Turbulence before the Final Period of Decay for Four-Point Correlation in a Rotating System for First-Order Reactant." American Journal of Theoritical and Applied Statistics, Vol. 3, Issue 4, pp. 81-89, 2014.

[12] Rahaman, M. L., "Decay of first order reactant in incompressible MHD turbulence before the final period for the case of multi-point and multi-time in a rotating system." J. Mech. Cont. \& Math. Sci. Vol. 4(2), pp. 509-522, 2010.

[13] Sarker, M. S. A. and N. Kishore, "Decay of the MHD turbulence before the final period." Intenational Journal of Engineering Science, Vol. 29(11), pp. 1479-1485, 1991. 
[14] Shebalin, J. V., "Ideal homogeneous magnetohydrodynamic turbulence in the presence of rotation and a mean magnetic field." Journal of Plasma Physics, Vol. 72, part 4, pp. 507-524, 2006.
[15] Shebalin, J. V., "The statistical mechanics of ideal homogeneous turbulence." NASA TP-2002-210783, NASA JSC, Houston, TX, 2002. 